\title{
Use of high-resolution 3.0-T magnetic resonance imaging to characterize atherosclerotic plaques in patients with cerebral infarction
}

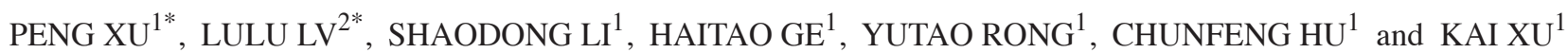 \\ ${ }^{1}$ Department of Radiology, Affiliated Hospital of Xuzhou Medical College, Xuzhou, Jiangsu 221006; \\ ${ }^{2}$ Department of Computed Tomography and Magnetic Resonance Imaging, \\ Xuzhou Central Hospital, Xuzhou, Jiangsu 221009, P.R. China
}

Received November 12, 2014; Accepted September 1, 2015

DOI: $10.3892 /$ etm.2015.2815

\begin{abstract}
The present study aimed to evaluate the utility of high-resolution magnetic resonance imaging (MRI) in the characterization of atherosclerotic plaques in patients with acute and non-acute cerebral infarction. High-resolution MRI of unilateral stenotic middle cerebral arteries was performed to evaluate the degree of stenosis, the wall and plaque areas, plaque enhancement patterns and lumen remodeling features in 15 and 17 patients with acute and non-acute cerebral infarction, respectively. No significant difference was identified in the vascular stenosis rate between acute and non-acute patients. Overall, plaque eccentricity was observed in 29 patients, including 13 acute and 16 non-acute cases, with no significant difference identified between these groups. The wall area of stenotic arteries and the number of cases with plaque enhancement were significantly greater in the acute patients, but no significant difference in plaque or lumen area was identified between the 2 patient groups. Lumen remodeling patterns of stenotic arteries significantly differed between the acute and non-acute patients; the former predominantly demonstrated positive remodeling, and the latter group demonstrated evidence of negative remodeling. In conclusion, patients with acute and non-acute cerebral infarction exhibit specific characteristics in stenotic arteries and plaques, which can be effectively evaluated by high-resolution MRI.
\end{abstract}

Correspondence to: $\mathrm{Mr}$. Kai Xu, Department of Radiology, Affiliated Hospital of Xuzhou Medical College, 99 Huaihai West Road, Xuzhou, Jiangsu 221006, P.R. China

E-mail: xuk_xuzhou@126.com

${ }^{*}$ Contributed equally

Key words: stroke, atherosclerosis, plaque, high resolution, magnetic resonance imaging

\section{Introduction}

Cerebral atherosclerosis is an important risk factor for ischemic stroke, and is the cause of stroke in $8-15 \%$ of patients (1). Factors that lead to stroke and that are associated with cerebral artery stenosis include intraluminal embolus, perforating artery occlusion and low perfusion (2). Currently, common techniques used for cerebrovascular evaluation include computed tomography angiography, magnetic resonance angiography (MRA) and digital subtraction angiography (DSA); however, the majority of these only examine the vascular lumen. With the development of interventional vascular therapy, pre-operative evaluation for interventional treatment of cerebrovascular diseases should not be limited to the location and stenosis of afflicted vessels. Rather, it is also important to determine the distribution, shape and properties of plaques on stenotic artery walls in order to properly evaluate surgical procedures and patient prognoses. High-resolution magnetic resonance imaging (HR-MRI) is a novel technique developed to examine blood vessels, specifically in the evaluation of cerebral vessel wall plaques (3). Previous HR-MRI studies demonstrated that vascular lumen morphology was significantly different between patients with symptomatic and asymptomatic cerebral infarction (4). However, vessel wall morphology and plaque enhancement patterns during different periods of cerebral infarction have not yet been reported. In the present study, 3.0-T HR-MRI was performed to investigate the morphological characteristics of afflicted vessels during different periods of cerebral infarction in patients with acute and non-acute cerebral infarction.

\section{Materials and methods}

Clinical data. Informed consent was obtained from all patients prior to an MRI scan. The study was approved by the institutional review board and ethics commission of the Affiliated Hospital of Xuzhou Medical College (Xuzhou, China), and 32 patients were prospectively included. Clinical data were collected from patients with unilateral middle cerebral artery (MCA) stenosis confirmed by brain MRA, which was performed in the Affiliated Hospital of Xuzhou Medical College between November 2013 and April 2014. Patients with any of the following conditions 
were excluded: An age of $<50$ years; non-atherosclerotic cerebrovascular disease, including vascular malformations, arterial dissection and arteritis; $<50 \%$ MCA stenosis; and HR-MRI of insufficient quality to meet measurement requirements.

HR-MRI examination. Time-of-flight (TOF) MRA was performed using a Discovery MR750 3.0-T superconducting MRI scanner (GE Healthcare, Piscataway, NJ, USA) equipped with a 16-channel phased array coil.

Scan parameters were as follows: Repetition time (TR)/echo time (TE), 27/6.9 msec; field of view (FOV), 24x16 cm; layer thickness, $1.6 \mathrm{~mm}$; flip angle, 20 number of excitations (NEX), 1; and matrix size, 320x256. For diffusion-weighted imaging (DWI) these parameters were as follows: TR/TE, 4,500/90 msec; FOV, 24x24 cm; layer thickness, $6.0 \mathrm{~mm}$; and NEX, 3 .

For HR-MRI, proton density-weighted imaging (PDWI) scan parameters were as follows: TR/TE, 2,900/20 msec; FOV, 12x12 cm; layer thickness, $2.5 \mathrm{~mm}$; NEX, 4; echo train length (ETL), 10; matrix size, 256x256; and voxel size, $0.22 \times 0.22 \times 2.5 \mathrm{~mm}$. For T1WI and enhanced T1WI, the parameters were as follows: TR/TE, 600/12 msec; FOV, 12x12 cm; layer thickness, 2.5 mm; NEX, 4; ETL, 3; matrix size, 256x256; and voxel size, $0.22 \times 0.22 \times 2.5 \mathrm{~mm}$.

Contrast-enhanced T1-weighted images were captured 2 min after intravenous injection of gadopentetate dimeglumine (Magnevist; Bayer Schering Pharma AG, Leverkusen, Germany) at $0.1 \mathrm{mmol} / \mathrm{kg}$ of body weight. A total of 6 slices were imaged in each HR-MRI sequence, and the scan time was 2-3 min for each.

Image analysis. All images were uploaded to an AW Volume Share 5 (4.6) workstation (GE Healthcare) for analysis. Image quality was rated on a scale of 1-5 (in which a score of 5 indicated excellent image quality). Parameter measurement and quantitative analysis were conducted on images with a score of $\geq 3$ (5). Patients were classified into the acute and non-acute cerebral infarction (including no infarction and old infarction) groups based on the presence or absence of high DWI signal intensity. The outer wall boundary and the vessel lumen of the narrowest vessel segments were manually drawn using the PDWI sequences (Fig. 1), and lumen and vessel areas were automatically calculated by the workstation software. Wall area $=$ vessel area - lumen area. A normal layer at the proximal end of the lesion was selected as the reference layer; if there was no suitable layer at the proximal end, a normal layer at the distal end was selected. Stenosis rate $=(1-$ lumen area of the narrowest layer / lumen area of the reference layer) x 100; remodeling rate $=$ vessel area of the narrowest layer / vessel area of the reference layer. A remodeling rate of $\leq 0.95$ was defined as negative remodeling (NR), and a rate of $\geq 1.05$ was considered positive remodeling (PR) (6). An eccentric plaque was defined as localized plaque surrounding $<75 \%$ of the vessel wall. Image quality and target vessels were analyzed by two experienced imaging physicians, with 16 and 14 years of experience, in a blinded manner, and a consensus was achieved through discussion when disagreements occurred.

Statistical analysis. All data were analyzed using SPSS 13.0 (SPSS Inc., Chicago, IL, USA). The basic clinical data, stenosis

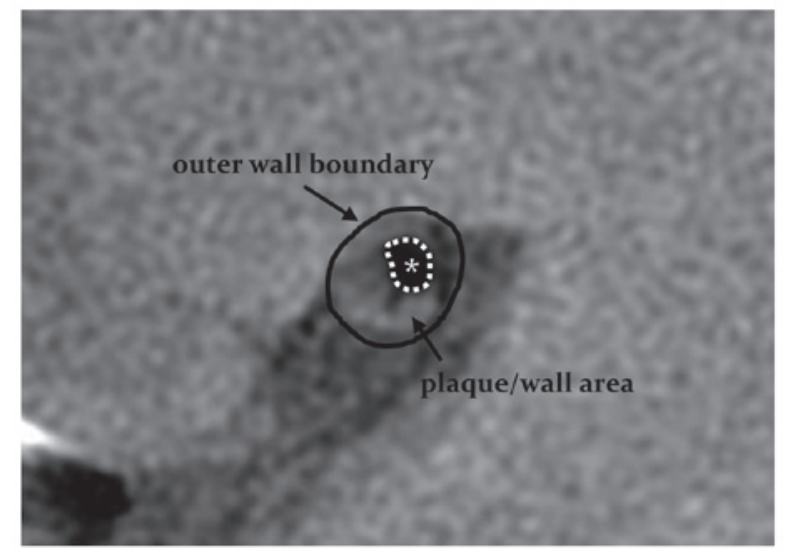

Figure 1. Methodology of plaque/wall area measurements. The solid black line delineates the outer wall boundary and the dotted white line, with asterisk, indicates the vessel lumen area.

rate, wall area, lumen area, plaque area, plaque enhancement patterns and wall remodeling rate were compared between the acute and non-acute infarction groups. Measurement data were expressed as the mean \pm standard deviation and compared between the two groups using a two-sample Student's t-test. Enumerated data were expressed as frequency and percentage and compared between the two groups using $\chi^{2}$ or Fisher's exact tests. $\mathrm{P}<0.05$ was considered to indicate a statistically significant difference.

\section{Results}

A total of 32 patients met the inclusion criteria, including 15 acute and 17 non-acute cerebral infarction patients. The time from onset to MRI ranged from $6 \mathrm{~h}$ to 3 days for acute patients. The patients' general information and atherosclerosis risk factors are listed in Table I. The quality of all images met the aforementioned quantitative analysis requirements. There was no significant difference in image quality between the DWI-positive and -negative groups (3.5 vs. 3.6; $\mathrm{P}>0.05$ ).

Overall, eccentric plaques were observed in 29 patients, including 13 acute and 16 non-acute infarction patients. The vascular stenosis rates were $70.12 \pm 16.42$ and $68.22 \pm 12.87 \%$ in the acute and non-acute patients, respectively $(\mathrm{P}=0.281)$. The acute group was significantly different from the non-acute group with regard to wall area, lumen remodeling rate and plaque enhancement $(\mathrm{P}=0.002, \mathrm{P}=0.002$ and $\mathrm{P}=0.004$, respectively). The wall area of stenotic segments was significantly greater in the acute group compared with the non-acute group $\left(11.84 \pm 2.81\right.$ vs. $8.75 \pm 2.02 \mathrm{~mm}^{2}$; $\mathrm{P}=0.002)$. No significant difference was observed in lumen area $\left(3.24 \pm 1.38\right.$ vs. $\left.3.05 \pm 1.42 \mathrm{~mm}^{2} ; \mathrm{P}=0.873\right)$ and plaque area $\left(6.44 \pm 2.61\right.$ vs. $\left.5.61 \pm 2.17 \mathrm{~mm}^{2} ; \mathrm{P}=0.257\right)$ between the acute and non-acute groups. Plaque enhancement was observed in 12 acute and 4 non-acute infarction patients $(\mathrm{P}=0.004$; Table II; Figs. 2 and 3).

\section{Discussion}

Cerebral atherosclerosis is a common disease, and plaque formation and rupture are important factors in the pathogenesis 
Table I. Demographic data.

\begin{tabular}{lccr}
\hline Demographic group & DWI $(+)(\mathrm{n}=15)$ & DWI(-) $(\mathrm{n}=17)$ & P-value \\
\hline Age, years $^{\mathrm{a}}$ & $65.2 \pm 14.8$ & $66.4 \pm 11.9$ & 0.614 \\
Male, n (\%) & $8(53.3)$ & $7(41.2)$ & 0.723 \\
Hypertension, n (\%) & $13(86.7)$ & $12(70.6)$ & 0.402 \\
Diabetes mellitus, n (\%) & $5(33.3)$ & $4(23.5)$ & 0.699 \\
Smoking, n (\%) & $6(40.0)$ & $5(29.4)$ & 0.712 \\
Hypercholesterolemia, n (\%) & $10(66.7)$ & $9(52.9)$ & 0.491 \\
Cardiovascular history, n (\%) & $5(33.3)$ & $7(41.2)$ & 0.726
\end{tabular}

${ }^{\mathrm{a}}$ Mean \pm standard deviation. DWI, diffusion-weighted imaging.

Table II. Comparison of stenotic arteries between acute and non-acute cerebral infarction.

\begin{tabular}{|c|c|c|c|}
\hline Features & $\operatorname{DWI}(+)(\mathrm{n}=15)$ & DWI(-) $(\mathrm{n}=17)$ & P-value \\
\hline Stenosis degree, $\%$ & $70.12 \pm 16.42$ & $68.22 \pm 12.87$ & 0.281 \\
\hline Wall area, $\mathrm{mm}^{2}$ & $11.84 \pm 2.81$ & $8.75 \pm 2.02$ & 0.002 \\
\hline Lumen area, $\mathrm{mm}^{2}$ & $3.24 \pm 1.38$ & $3.05 \pm 1.42$ & 0.873 \\
\hline Plaque area, $\mathrm{mm}^{2}$ & $6.44 \pm 2.61$ & $5.61 \pm 2.17$ & 0.257 \\
\hline Eccentric plaque, n (\%) & $13(86.7)$ & $16(94.1)$ & 0.589 \\
\hline Plaque enhancement, n (\%) & $12(80.0)$ & $4(23.5)$ & 0.004 \\
\hline Remodeling rate $^{\mathrm{a}}$ & $1.08 \pm 0.19$ & $0.87 \pm 0.14$ & 0.002 \\
\hline
\end{tabular}

${ }^{\mathrm{a}}$ Mean \pm standard deviation. DWI, diffusion-weighted imaging.

of ischemic stroke and transient ischemic attack (TIA). Previous carotid artery studies have indicated that MRI could effectively evaluate carotid artery plaque characteristics (7). Due to the small lumen of cerebral arteries and the limitations of MRI, only a few studies have evaluated cerebral arterial walls and plaques $(3,8)$, but the development of HR-MRI has made it possible to assess intracranial arterial wall morphology using MRI.

Klein et al (9) demonstrated that HR-MRI could produce images of cerebral arterial walls and lumina; in specific cases in which conventional TOF MRA provides normal findings or images of a rough wall, HR-MRI can detect wall plaques that are not visible using MRA. This is hypothesized to be associated with vascular lumen remodeling. Early studies investigating the coronary arteries identified two patterns of lumen remodeling: PR and NR. The former is characterized by outward expansion of blood vessels, which aids in maintaining vascular lumen size, but makes the plaques vulnerable to rupture, thus causing TIA and acute stroke. The latter is characterized by inward blood vessel contraction, which can aggravate stenosis, but allows the plaques to be relatively stable $(9,10)$. Thus, a large wall plaque may not cause stenosis due to vascular lumen remodeling. For this reason, traditional TOF MRA and DSA have certain limitations and can be misleading when evaluating stenosis or risk factors. Li et al (11) performed TOF MRA and HR-MRI examinations in 48 patients with suspected MCA stenosis. The study reported that HR-MRI detected abnormal vessel walls that were diagnosed as normal by TOF MRA in 5 patients, and 2 patients were diagnosed with vascular stenosis using TOF MRA, but no abnormal lumen was reported by HR-MRI. These findings were considered to be associated with the different remodeling patterns in the stenotic lumen.

In the present study, the acute cerebral infarction group had a wall area significantly greater than that of the non-acute group, and predominantly exhibited PR. No significant differences were identified in lumen area or plaque area, and therefore, it was suggested that the presence or absence of increased DWI signal intensity was due to vascular remodeling. In previous studies, the remodeling pattern of stenotic arteries has been associated with interventional surgery complications (12); patients with NR may be at greater risk of injury during interventional therapy, and pre-operative HR-MRI aids in the assessment of the risk of surgery (13).

HR-MRI is currently the best way to evaluate cerebral artery plaque stability. Due to the limited resolution of MRI equipment, cerebral arterial plaque composition analysis remains in the exploratory stage (14). Conversely, the analysis of carotid atherosclerotic plaque composition using HR-MRI is relatively mature, and intracranial atherosclerotic plaques have properties similar to those of carotid atherosclerotic plaques (15).

Unstable plaques are characterized by a large intraplaque lipid core or intraplaque hemorrhage, and a thin or discontinuous fibrous cap, whereas stable plaques are mainly composed of fiber, have a small or no lipid core, and a thick and continuous fibrous cap $(16,17)$. Plaque enhancement, in addition to signal 
A

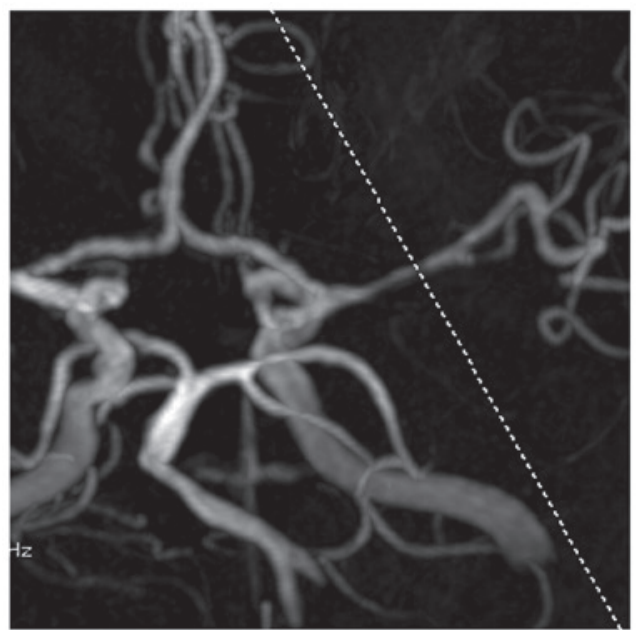

B

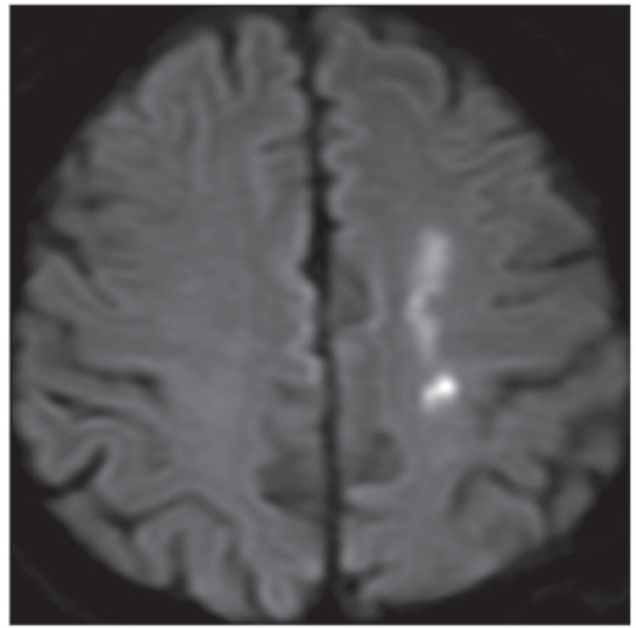

C

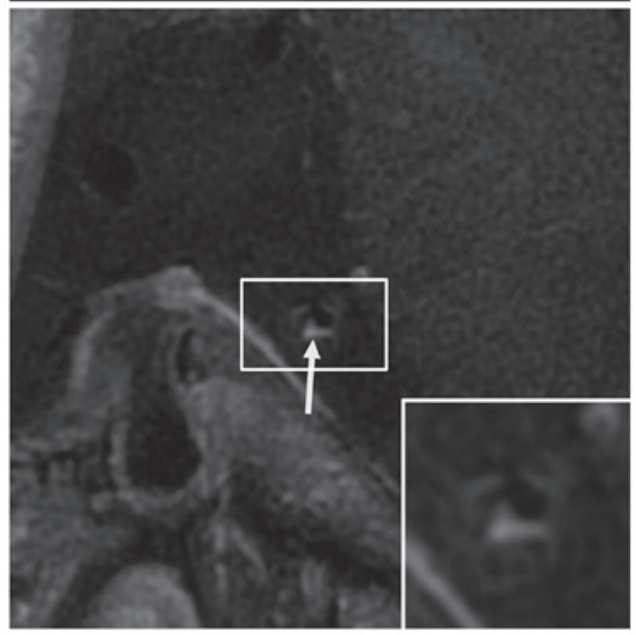

Figure 2. Magnetic resonance angiography (MRA) and high-resolution magnetic resonance imaging (HR-MRI) results of a 68 -year-old male patient with acute cerebral infarction. (A) MRA revealed stenosis of the M1 segment of the left middle cerebral artery; (B) Diffusion-weighted imaging revealed a patchy high signal in the left centrum semiovale; and (C) HR-MRI demonstrated eccentric plaques with significant enhancement (arrow). The remodeling rate of the stenotic lumen was 1.12 .

characteristics, is associated with plaque stability. Enhanced imaging reveals enhanced wall plaques of afflicted arteries in acute stroke patients and plaque enhancement is associated with the infarction distribution $(18,19)$. In the present study, wall plaque enhancement was observed in 12 and 4 patients in the DWI-positive and -negative groups, respectively.
A

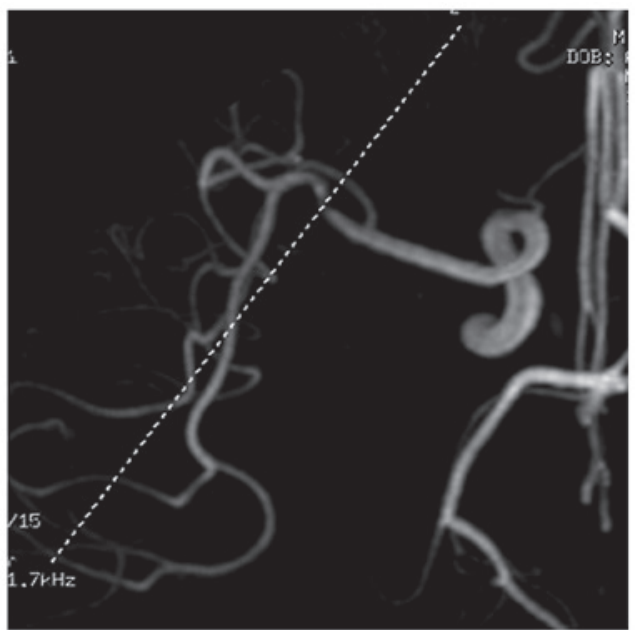

B

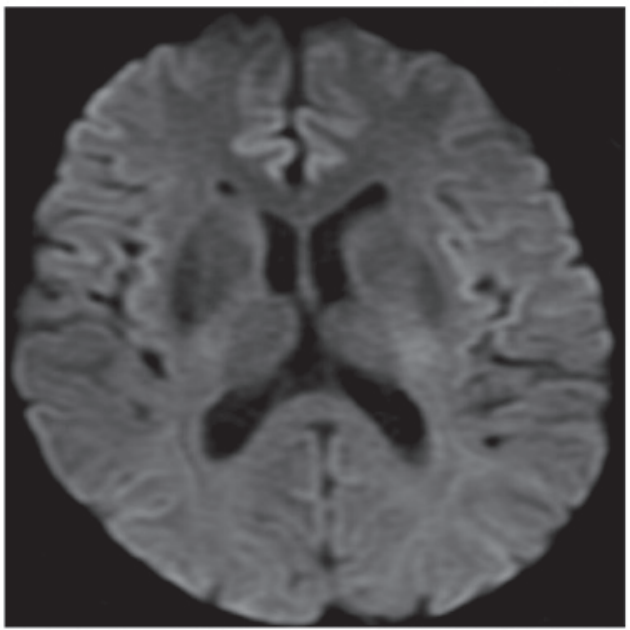

C

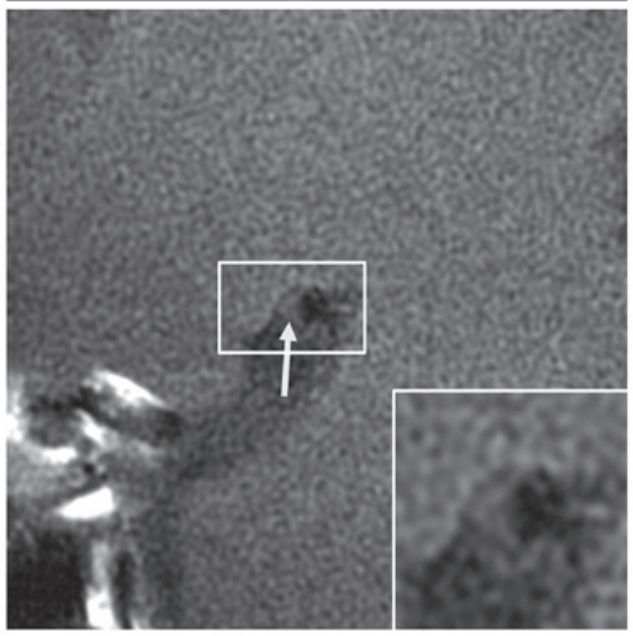

Figure 3. Magnetic resonance angiography (MRA) and high-resolution magnetic resonance imaging (HR-MRI) results of a 64-year-old male patient with non-acute cerebral infarction. (A) MRA revealed marked stenosis of the distal M1 segment of the right middle cerebral artery; (B) Diffusion-weighted imaging revealed no abnormal high signal; and (C) HR-MRI revealed no plaque enhancement (arrow). The remodeling rate of the stenotic lumen was 0.89 .

The plaque enhancement rate was significantly higher in the DWI-positive group, and wall plaque enhancement was considered to be associated with internal neovascularization or inflammatory reactions (20).

The present study has certain limitations. First, although HR-MRI is more effective than conventional TOF MRA in 
evaluating cerebral arterial wall plaques, the majority of the results are based on associated results of carotid or coronary plaques from earlier studies and it is difficult to obtain pathological results of cerebral artery plaques. For this reason, the evaluation of cerebral artery plaque morphology and properties is subjective to a certain extent. Second, due to the small total sample size, the cerebral infarction patients were divided into acute and non-acute groups based on DWI only, and were not further divided according to infarction time. Moreover, the severity of cerebral infarction in the acute patients was not clearly defined, meaning that there were a few uncertain factors in the stenotic artery analysis. Third, plaque enhancement was mainly determined based on the subjective judgment of the physicians; no quantitative analysis was performed. In future studies, quantitative analyses of plaque signal intensities prior to and following enhancement may aid in determining the association between plaque properties and cerebral infarction.

In conclusion, the present study showed that HR-MRI can provide high-quality images of vessel wall structure that cannot be achieved with conventional MRI, and thus can serve as a clear complement to TOF MRA to perform a more detailed analysis of stenotic artery walls in patients with cerebral infarction, including resolving plaque properties. HR-MRI can thus assist in clinical treatment and in determining patient prognosis.

\section{Acknowledgements}

This study was supported by funding from Xuzhou Medical College (grant no. 2014KJ17).

\section{References}

1. Wityk RJ, Lehman D, Klag M, Coresh J, Ahn H and Litt B: Race and sex differences in the distribution of cerebral atherosclerosis. Stroke 27: 1974-1980, 1996.

2. Wong KS, Gao S, Chan YL, Hansberg T, Lam WW, Droste DW, Kay R and Ringelstein EB: Mechanisms of acute cerebral infarctions in patients with middle cerebral artery stenosis: A diffusion-weighted imaging and microemboli monitoring study. Ann Neurol 52: 74-81, 2002.

3. Ryu CW, Jahng GH, Kim EJ, Choi WS and Yang DM: High resolution wall and lumen MRI of the middle cerebral arteries at 3 tesla. Cerebrovasc Dis 27: 433-442, 2009.

4. Xu WH, Li ML, Gao S, Ni J, Zhou LX, Yao M, Peng B, Feng F, Jin ZY and Cui LY: In vivo high-resolution MR imaging of symptomatic and asymptomatic middle cerebral artery atherosclerotic stenosis. Atherosclerosis 212: 507-511, 2010.
5. Yuan C, Mitsumori LM, Ferguson MS, Polissar NL, Echelard D, Ortiz G, Small R, Davies JW, Kerwin WS and Hatsukami TS: In vivo accuracy of multispectral magnetic resonance imaging for identifying lipid-rich necrotic cores and intraplaque hemorrhage in advanced human carotid plaques. Circulation 104: 2051-2056, 2001.

6. Ma N, Jiang WJ, Lou X, Ma L, Du B, Cai JF and Zhao TQ: Arterial remodeling of advanced basilar atherosclerosis: a 3-tesla MRI study. Neurology 75: 253-258, 2010.

7. Yuan C, Beach KW, Smith LH Jr and Hatsukami TS: Measurement of atherosclerotic carotid plaque size in vivo using high resolution magnetic resonance imaging. Circulation 98: 2666-2671, 1998.

8. Niizuma K, Shimizu H, Takada S and Tominaga T: Middle cerebral artery plaque imaging using 3-Tesla high-resolution MRI. J Clin Neurosci 15: 1137-1141, 2008.

9. Klein IF, Lavallée PC, Mazighi M, Schouman-Claeys E, Labreuche $\mathrm{J}$ and Amarenco P: Basilar artery atherosclerotic plaques in paramedian and lacunar pontine infarctions: A high-resolution MRI study. Stroke 41: 1405-1409, 2010.

10. Schoenhagen P, Ziada KM, Kapadia SR, Crowe TD, Nissen SE and Tuzcu EM: Extent and direction of arterial remodeling in stable versus unstable coronary syndromes: An intravascular ultrasound study. Circulation 101: 598-603, 2000.

11. Li ML, Xu WH, Song L, Feng F, You H, Ni J, Gao S, Cui LY and Jin ZY: Atherosclerosis of middle cerebral artery: Evaluation with high-resolution MR imaging at 3T. Atherosclerosis 204: 447-452, 2009.

12. Yamagishi M, Terashima M, Awano K, Kijima M, Nakatani S, Daikoku S, Ito K, Yasumura Y and Miyatake K: Morphology of vulnerable coronary plaque: Insights from follow-up of patients examined by intravascular ultrasound before an acute coronary syndrome. J Am Coll Cardiol 35: 106-111, 2000.

13. Jiang WJ, Yu W, Ma N, Du B, Lou X and Rasmussen PA: High resolution MRI guided endovascular intervention of basilar artery disease. J Neurointerv Surg 3: 375-378, 2011.

14. SwartzRH,BhutaSS,Farb RI,Agid R, Willinsky RA, Terbrugge KG, Butany J, Wasserman BA, Johnstone DM, Silver FL and Mikulis DJ: Intracranial arterial wall imaging using high-resolution 3-tesla contrast-enhanced MRI. Neurology 72: 627-634, 2009.

15. Fayad ZA and Fuster V: Characterization of atherosclerotic plaques by magnetic resonance imaging. Ann NY Acad Sci 902: 173-186, 2000.

16. Saam T, Cai J, Ma L, Cai YQ, Ferguson MS, Polissar NL, Hatsukami TS and Yuan C: Comparison of symptomatic and asymptomatic atherosclerotic carotid plaque features with in vivo MR imaging. Radiology 240: 464-472, 2006.

17. Saam T, Yuan C, Chu B, Takaya N, Underhill H, Cai J, Tran N, Polissar NL, Neradilek B, Jarvik GP, et al: Predictors of carotid atherosclerotic plaque progression as measured by noninvasive magnetic resonance imaging. Atherosclerosis 194: e34-e42, 2007.

18. Kim JM, Jung KH, Sohn CH, Moon J, Han MH and Roh JK: Middle cerebral artery plaque and prediction of the infarction pattern. Arch Neurol 69: 1470-1475, 2012.

19. Demarco JK, Ota H, Underhill HR, Zhu DC, Reeves MJ, Potchen MJ, Majid A, Collar A, Talsma JA, Potru S, et al: MR carotid plaque imaging and contrast-enhanced MR angiography identifies lesions associated with recent ipsilateral thromboembolic symptoms: An in vivo study at 3T. AJNR Am J Neuroradiol 31: 1395-1402, 2010.

20. Chen XY, Wong KS, Lam WW, Zhao HL and Ng HK: Middle cerebral artery atherosclerosis: Histological comparison between plaques associated with and not associated with infarct in a postmortem study. Cerebrovasc Dis 25: 74-80, 2008. 